\title{
Superior Colliculus Mediates Cervical Dystonia Evoked by Inhibition of the Substantia Nigra Pars Reticulata
}

\author{
Angela L. Holmes, ${ }^{1,2}$ Patrick A. Forcelli, ${ }^{1,2}$ Jacqueline T. DesJardin, ${ }^{1}$ Ashley L. Decker, ${ }^{1}$ Menna Teferra, ${ }^{1}$ \\ Elizabeth A. West, ${ }^{1,2}$ Ludise Malkova, ${ }^{1,2}$ and Karen Gale ${ }^{1,2}$ \\ ${ }^{1}$ Department of Pharmacology and Physiology and ${ }^{2}$ The Interdisciplinary Program in Neuroscience, Georgetown University Medical Center, Washington, \\ DC 20007
}

Cervical dystonia (CD; spasmodic torticollis) can be evoked by inhibition of substantia nigra pars reticulata (SNpr) in the nonhuman primate (Burbaud et al., 1998; Dybdal et al., 2012). Suppression of GABAergic neurons that project from SNpr results in the disinhibition of the targets to which these neurons project. It therefore should be possible to prevent CD by inhibition of the appropriate nigral target region(s). Here we tested the hypothesis that the deep and intermediate layers of the superior colliculus (DLSC), a key target of nigral projections, are required for the emergence of $\mathrm{CD}$. To test this hypothesis, we pretreated the DLSC of four macaques with the GABA agonist muscimol to determine whether this treatment would prevent CD evoked by muscimol infusions in SNpr. Our data supported this hypothesis: inhibition of DLSC attenuated CD evoked by muscimol in SNpr in all four animals. In two of the four subjects, quadrupedal rotations were evoked by muscimol application into SNpr sites that were distinct from those that induced dystonia. We found that inhibition of DLSC did not significantly alter quadrupedal rotations, suggesting that this response is dissociable from the SNpr-evoked CD. Our results are the first to demonstrate a role of DLSC in mediating the expression of CD. Furthermore, these data reveal a functional relationship between SNpr and DLSC in regulating posture and movement in the nonhuman primate, raising the possibility that the nigrotectal pathway has potential as a target for therapeutic interventions for CD.

\section{Introduction}

The basal ganglia are critical for the control of posture, motor coordination, initiation of movement, and procedural learning (Blandini et al., 2000). Dysfunction in basal ganglia output nuclei [substantia nigra pars reticulata (SNpr) and globus pallidus internal segment (GPi)] gives rise to motor and postural abnormalities, including akinesia, dyskinesia, and dystonia (Mink, 2003).

Motor and postural abnormalities have been evoked by inhibition of SNpr in experimental animals. One of these abnormal symptoms is cervical dystonia (CD), the most common form of focal dystonia, involving the head and neck (also called spasmodic torticollis). Although CD has never been evoked from the SNpr of the rat, it has been documented to occur after unilateral inhibition of SNpr in the nonhuman primate (Burbaud et al., 1998; Dybdal et al., 2012) and following unilateral electrolytic lesions of SNpr in cats (Malouin and Bédard, 1983). In contrast, inhibition of GPi does not induce CD (Burbaud et al., 1998), suggesting that a target of $\mathrm{SNpr}$ projections may be especially

\footnotetext{
Received May 11, 2012; revised July 13, 2012; accepted Aug. 5, 2012.

Author contributions: A.L.H., L.M., and K.G. designed research; A.L.H.,P.A.F., J.T.D., A.L.D.,M.T., E.A.W., L.M., and K.G. performed research; A.L.H., P.A.F., L.M., and K.G. analyzed data; A.L.H., P.A.F., J.T.D., E.A.W., L.M., and K.G. wrote the paper.

This research was supported by NIH Grants F31 NS061623 (A.L.H.), T32 NS041231 (A.L.H.), R01 NS020576 (K.G.), R21HD039937 (L.M.), and R01MH82364 (L.M.), and Howard Hughes Medical Institute Undergraduate Research Program (J.T.D). We thank Carrie Silver, Celeste Wallenta, Jadig Garcia, and Sandra Lopez for technical assistance. The authors declare no competing financial interests.

Correspondence should be addressed to Dr. Karen Gale, Department of Pharmacology, Georgetown University Medical Center, 3900 Reservoir Road NW, Washington, DC 20007. E-mail: galek@georgetown.edu.

DOI:10.1523/JNEUROSCI.2295-12.2012

Copyright $\odot 2012$ the authors $\quad 0270-6474 / 12 / 3213326-07 \$ 15.00 / 0$
}

important for the genesis of CD. While GPi and SNpr both project to the thalamus and pedunculopontine nucleus, the one projection target of SNpr not shared by GPi is the superior colliculus (Laursen, 1955; Nauta and Mehler, 1966; Beckstead et al., 1981; Beckstead and Frankfurter, 1982; Sidibé et al., 1997).

The deep and intermediate layers of the superior colliculus (DLSC) together constitute a principal projection target of the SNpr; this nigrotectal projection is GABAergic and tonically active (Hikosaka and Wurtz, 1985a,b; Niemi-Junkola and Westby, 1998). Thus, phasic pausing of nigral firing (triggered by physiological GABAergic input from striatum) results in decreased inhibition of DLSC (Hikosaka and Wurtz, 1985b; Redgrave et al., 1992; Niemi-Junkola and Westby, 1998). This state of disinhibition is permissive to neuronal activity resulting in movement. Consistent with this model, inhibition of DLSC has been shown to prevent stereotyped hyperactivity induced by inhibition of SNpr in the rat (Dean et al., 1982; Taha et al., 1982; Gunne et al., 1988). Thus, in the present study we tested the hypothesis that inhibition of the DLSC in the nonhuman primate would prevent CD induced by inhibition of SNpr.

To determine the functional interaction between SNpr and DLSC in the monkey, we focally infused muscimol (MUS), a $\mathrm{GABA}_{\mathrm{A}}$ receptor agonist, into SNpr and DLSC. Inhibition of SNpr normally results from the activity of GABAergic projections coming from globus pallidus external segment and striatum; these in turn silence the nigral GABA projections to DLSC, thalamus, and pedunculopontine nucleus, thereby activating (disinhibiting) these targets. As an experimental manipulation, the application of MUS focally into SNpr effectively inhibits all of the 
GABAergic SNpr output neurons in the vicinity of the drug infusion, including those projecting to DLSC. To selectively prevent activation of DLSC in our experiments, we pretreated the DLSC with MUS before MUS infusions into SNpr. We predicted that pharmacological inhibition of the DLSC with MUS would prevent the CD evoked by intranigral MUS only if the DLSC is a crucial target for the expression of this abnormality.

\section{Materials and Methods}

\section{Subjects}

Four rhesus macaques (Macaca mulatta), one female (ST) and three males (DE, OL, and DA), were used in this study. Animals were 3-5 years of age and weighed $4.4-7.0 \mathrm{~kg}$ at the beginning of this study. Monkeys were housed in pairs in a room with regulated lighting $(12 \mathrm{~h}$ light/dark cycle) and maintained on primate LabDiet (no. 5049; PMI Nutrition International) supplemented with fresh fruit/vegetables. Water was available ad libitum in the home cage. This study was conducted under a protocol approved by the Georgetown University Animal Care and Use Committee and in accordance with the Guide for Care and Use of Laboratory Animals adopted by the National Institutes of Health.

Each monkey was implanted with a stereotaxically positioned chronic infusion chamber, which allowed a removable injector, fitted with an infusion cannula of adjustable length, to be inserted into predetermined sites in the brain.

\section{Infusion chamber and injector}

The chamber and injector are described in detail by West et al. (2011) and Wellman et al. (2005). Briefly, a rectangular chamber (38 mm length $\times$ $44 \mathrm{~mm}$ width $\times 18 \mathrm{~mm}$ depth) made of polyoxymethylene (Helm Tech Machining) was implanted during an aseptic surgical procedure as described previously (West et al., 2011). A removable grid allowed injections spaced at $2 \mathrm{~mm}$ intervals in the anteroposterior and mediolateral planes. Adjustments to the length of the injection cannula set the dorsoventral position of the infusions.

The cannula was inserted using a custom-built telescoping injector (Elmeco Engineering; Wellman et al., 2005), allowing for the 27 ga stainless steel infusion cannula to be positioned at the desired intracerebral sites.

\section{Magnetic resonance imaging}

Postoperatively, each monkey received at least one T1-weighted scan to determine and/or verify the coordinates for the infusion sites. Additional scans were performed throughout the experiment as needed. Scans were conducted as described previously (West et al., 2011) with an effective resolution of $1.0 \times 1.0 \times 1.0 \mathrm{~mm}^{3}$. To verify the location of the predetermined infusion sites, tungsten microelectrodes (FHC) were inserted through the infusion grid. The tip of the electrode was placed $\sim 10 \mathrm{~mm}$ above the intended site and visualized on the MRI scan. Based on the position of the tip of the electrode on the MRI scan, the final coordinates for the drug infusions were adjusted with respect to the coordinates of the infusion grid.

\section{Intracerebral microinfusions}

As shown in Figures 1 and 2, two brain structures, DLSC and SNpr, respectively, were targeted by the infusions.

MUS ( $\mathrm{GABA}_{\mathrm{A}}$ agonist, $9 \mathrm{nmol}$ in $1 \mu \mathrm{l}$ ), bicuculline methiodide (BIC; $\mathrm{GABA}_{\mathrm{A}}$ antagonist, $10 \mathrm{nmol}$ in $\left.1 \mu \mathrm{l}\right)$, or sterile saline $(1 \mu \mathrm{l}$ of $0.9 \% \mathrm{NaCl}$ solution) were infused into the brain of the awake monkey while the animal was seated in a nonhuman primate chair (Crist Instrument Company) with minimum restraint. MUS was used to transiently suppress synaptic activity in the vicinity of the focal infusion site in DLSC and SNpr, while BIC was used to disinhibit (activate) DLSC. The drugs (as sterile solutions) were infused in a volume of $1 \mu \mathrm{l}$ at a rate of $0.2 \mu \mathrm{l} / \mathrm{min}$. Infusions were conducted using a pump-driven syringe connected by polyethylene tubing attached to the cannula, as described previously (West et al., 2011). When infusions were made into more than one site (i.e., for the SNpr-DLSC interaction experiments), infusions into DLSC were given before the unilateral infusion into SNpr. Bilateral infusions into DLSC were done simultaneously. DLSC infusions lasted 3-13 min.
The length of time between the completion of the bilateral DLSC infusions and the beginning of the unilateral SNpr infusion was $0-4 \mathrm{~min}$. The SNpr infusion lasted 3-8 min. The entire infusion procedure lasted $\sim 17$ $\mathrm{min}$. At least $48 \mathrm{~h}$ were allowed to elapse between experimental sessions in an individual subject.

\section{Diffusion of drugs and localization of drug effects}

The dose of MUS $(9 \mathrm{nmol} / 1 \mu \mathrm{l})$ used in our present study has been previously documented to affect a $3 \mathrm{~mm}$ diameter sphere in the rat brain (Martin, 1991; Martin and Ghez, 1999; Allen et al., 2008). Furthermore, we have previously documented the efficacy, time course, and site specificity of this dose of MUS in the SNpr of the nonhuman primate for evoking dystonia (Dybdal et al., 2012).

The dose of bicuculline $(10 \mathrm{nmol} / 1 \mu \mathrm{l})$ used in our present study is similar to that employed by Yoshida et al. (1991), who injected tritiated bicuculline methyl chloride into the striatum of rats and measured the area of diffusion. They found that a sphere of tissue of about $3 \mathrm{~mm}$ in diameter was affected. Thus, we expected that our BIC infusions would effectively disinhibit most of the area within DLSC.

\section{Experimental design}

MUS in SNpr. To identify sites that evoked rapid onset CD, we tested multiple sites. Microinfusions were performed in a total of 13 sites within the SNpr.

MUS was infused unilaterally into SNpr (4-31 infusions per animal), and the resulting behaviors were analyzed. Head tilt was analyzed separately from the co-occurring body lean to document and examine both components of the dystonic behavior. Sites determined to evoke dystonia were then used for SNpr-DLSC interaction experiments as described below. Saline $(1 \mu \mathrm{l})$ was also infused into effective sites to control for nonspecific effects of microinfusions. Each monkey received 1-2 control infusions in the effective sites.

MUS in DLSC. To determine the behavioral effects of MUS in DLSC, we infused MUS unilaterally (2-4 infusions per animal) and bilaterally (1-2 infusions in three animals: ST, DE, and DA) into DLSC and analyzed resulting behaviors. Saline $(1 \mu \mathrm{l})$ was infused into effective sites to control for nonspecific effects of microinfusions. Each animal received 1-2 control infusions in the effective sites.

Intracerebral infusions to examine SNpr-DLSC interactions. To test the hypothesis that pharmacological inhibition of DLSC with MUS would prevent CD evoked by MUS in SNpr, we pretreated DLSC with MUS before MUS infusion in SNpr. Three animals (ST, DE, and DA) received 2-4 bilateral infusions of MUS in DLSC immediately followed by a unilateral infusion of MUS in SNpr. Three monkeys (ST, DA, and OL) received infusions of MUS in DLSC immediately followed by a unilateral infusion of MUS in SNpr. Saline ( $1 \mu \mathrm{l}$ ) infusions into DLSC before MUS in SNpr were used as a control for nonspecific effects in DLSC. Each monkey received 1-4 control infusions in the effective sites.

BIC in DLSC. To determine whether activation of DLSC was sufficient to evoke abnormal postures and motor movements, we infused BIC in DLSC. Unilateral microinfusions of BIC were performed in a total of 10 sites within the DLSC (2-4 sites per animal).

BIC was infused unilaterally into DLSC (6-14 infusions per animal), and the behaviors were analyzed. Saline $(1 \mu \mathrm{l})$ was infused into the sites to control for nonspecific effects of microinfusions. Each monkey received 1-2 control infusions in the effective sites.

\section{Evaluation of abnormal postures and motor movements and baseline behavior}

Animals were videotaped in an observation cage for 30 min before infusion (baseline behavior) and for a minimum of 60 min following infusions. Animals were continuously monitored until behaviors returned to baseline levels. CD tended to last about $2 \mathrm{~h}$. In some cases, the behavior lasted as long as $4 \mathrm{~h}$; in these cases, animals were observed in the observation cage for a maximum of $2 \mathrm{~h}$ and then returned to the home cage and observed until the behavior was no longer present.

\section{Analyses of videotapes}

The baseline and postinfusion videotapes were coded and analyzed by two observers (one of whom was blind with respect to the treatment of 
the monkey) using Observer software (versions 5.0 and 9.0, Noldus). The videotapes were analyzed in 15 min segments (bins); two segments were obtained per baseline $(0-15$ and 15-30 $\mathrm{min})$ and four segments per infusion $(0-15,15-30,30-45$, and $45-60 \mathrm{~min})$.

Behaviors were coded as either events (number of occurrences of a particular behavior per 15 min bin) or states (duration of time during which the behaviors were present per 15 min bin). Of the behaviors that were scored, head tilt, body lean, and head turning were coded as states; quadrupedal rotations, eye saccades, limb dyskinesias, and lip/mouth twitch were coded as events. Observer(s) also noted general postures, use of limbs, activity level, and ability to engage in normal behaviors such as reaching for and eating treats.

Interobserver reliability was assessed by comparing the results of video segments scored by both observers (one-third of the experimental video records). Interobserver reliability was very high for both head tilt (Pearson's $r=$ 0.9932 ) and body lean (Pearson's $r=0.9590)$.

\section{Statistical analysis}

For analysis of site-specific effects of the drug infusions, we used only the first $30 \mathrm{~min}$ of the postinfusion data to capture the effects during the peak concentration of the drug within the intended target. Statistical analyses were performed using SPSS (version 20, IBM) and GraphPad Prism (version 5, GraphPad Software). Data were analyzed using a one-way ANOVA with infusion type as a repeated measure, followed by HolmSidak corrected one-tailed post hoc tests. Statistical significance was set at $p<0.05$.

\section{Results}

\section{Infusion site verification}

A detailed histological analysis was performed on one monkey (DE) to validate the accuracy of the MRI-based calculation of infusion sites. Each section through the SNpr and DLSC was mounted, defatted, stained with thionin, and coverslipped. As we have shown previously (Dybdal et al., 2012; Wellman et al., 2005), placement of the cannula localized in the histological sections closely corresponded with the placement determined from MRI. Photomicrographs of representative sections showing cannulae in DLSC and SNpr are presented in Figures $1 C$ and $2 C$, respectively. The histological analysis showed close coregistration with the MRI scan (animal DE; Figs. $1 B$ and $2 B$ ). The remaining three animals (ST, DA, and OL) were unavailable for histological processing because they were undergoing additional behavioral testing at the conclusion of the study. For these animals we evaluated the cannula placement based on the MRI scan.

\section{CD evoked from $\mathrm{SNpr}$}

Microinfusions of MUS evoked a rapid onset, contralaterally directed head tilt from at least one SNpr infusion site in each animal (Fig. 2 and Table 1). The latency to onset was between 0 and $13 \mathrm{~min}$ with a mean of $5.5 \mathrm{~min}( \pm 1.3 \mathrm{SEM})$. Two different types of dystonic

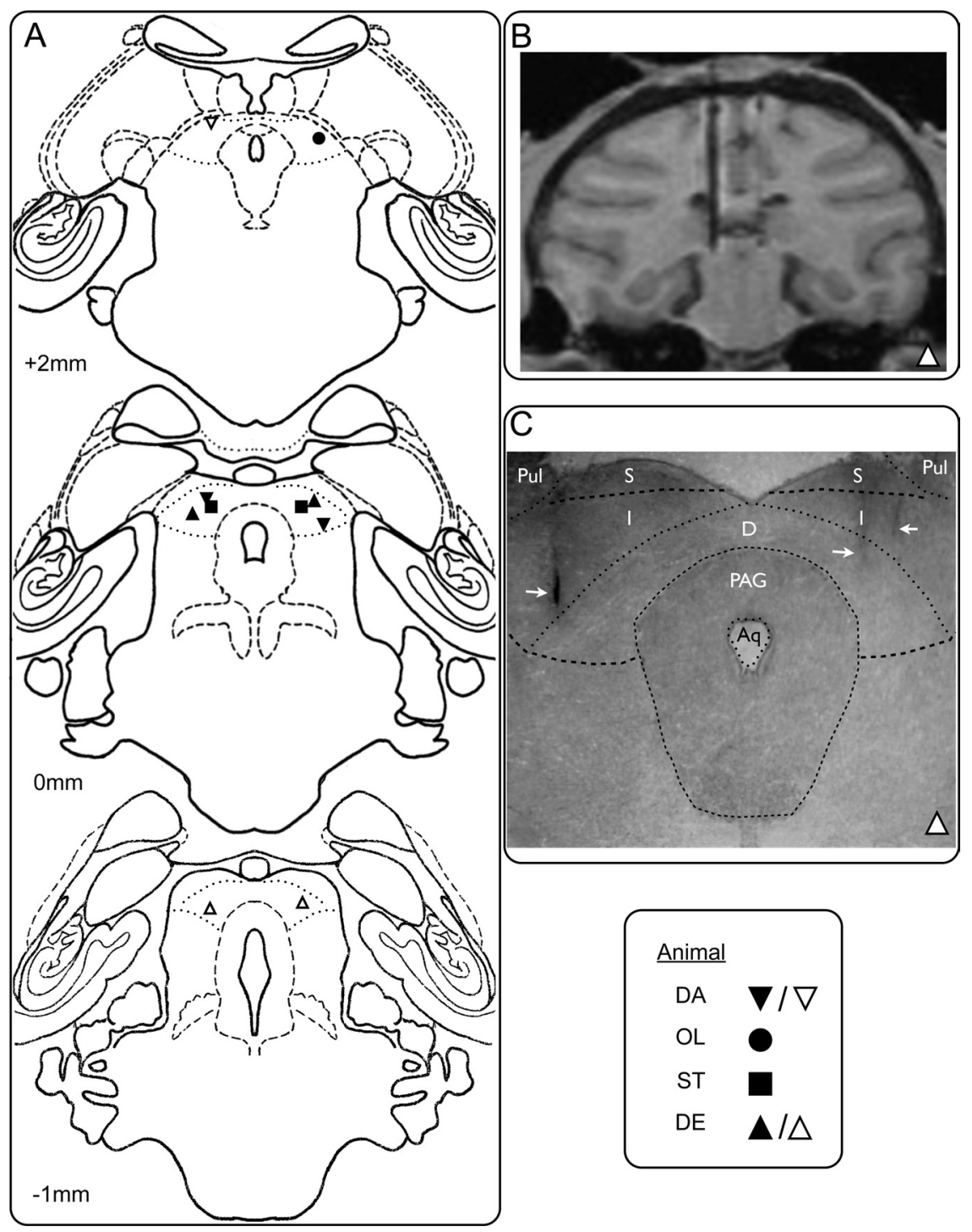

Figure 1. Histological verification of infusion sites in DLSC. $A$, Drawings of coronal sections from a standard macaque brain showing the placement of cannulae in DLSC. Numbers $(+2,0,-1)$ indicate the distance in millimeters from the interaural plane. Geometrical symbols show the placement of infusion sites determined from MRI (animals DA, OL, ST) or histology (DE). Black symbols represent sites used to examine SNpr-DLSC interactions. Clear symbols represent additional sites in which BIC was infused. B, MRI section through the brain of animal DE showing an electrode placed in the left DLSC. C, Photomicrograph of a histological section through DLSC in the same animal (DE) at the level corresponding to the MRI section in $\boldsymbol{B}$ and to the drawing in $\boldsymbol{A}$ at the level $0 \mathrm{~mm}$. White arrow on the left shows trace of the cannula placement corresponding to the electrode in $\boldsymbol{B}$. White arrows on the right show additional cannula traces. Pul, Pulvinar; $S$, superficial layers of the superior colliculus; I, intermediate layers of the superior colliculus; $D$, deep layers of the superior colliculus, PAG, periaqueductal gray; Aq, cerebral aqueduct. Dotted lines show anatomical boundaries.

head tilt were evoked: laterocollis (in animals DA, DE, and ST) and rotational collis (in animal OL). Laterocollis was characterized by a lateral flexion and a slight rotation of the neck, resulting in dystonic leaning of the head toward the shoulder; the face remained oriented forward and slightly downward, and the body remained upright. Between 2 and $17 \mathrm{~min}$ following the onset of the head tilt, the laterocollis reached maximum severity: the head was tilted horizontally and pressed against the shoulder contraversive to the side of microinfusion, with the body leaning in the direction of the head tilt. The body lean always occurred after the laterocollis reached maximum severity. Rotational collis was characterized by the body and head remaining largely upright, but with the head rotated in one direction (contraversive to the side of microinfusion) with the face turned to the same side. As the behavior progressed over time (between 6 and 


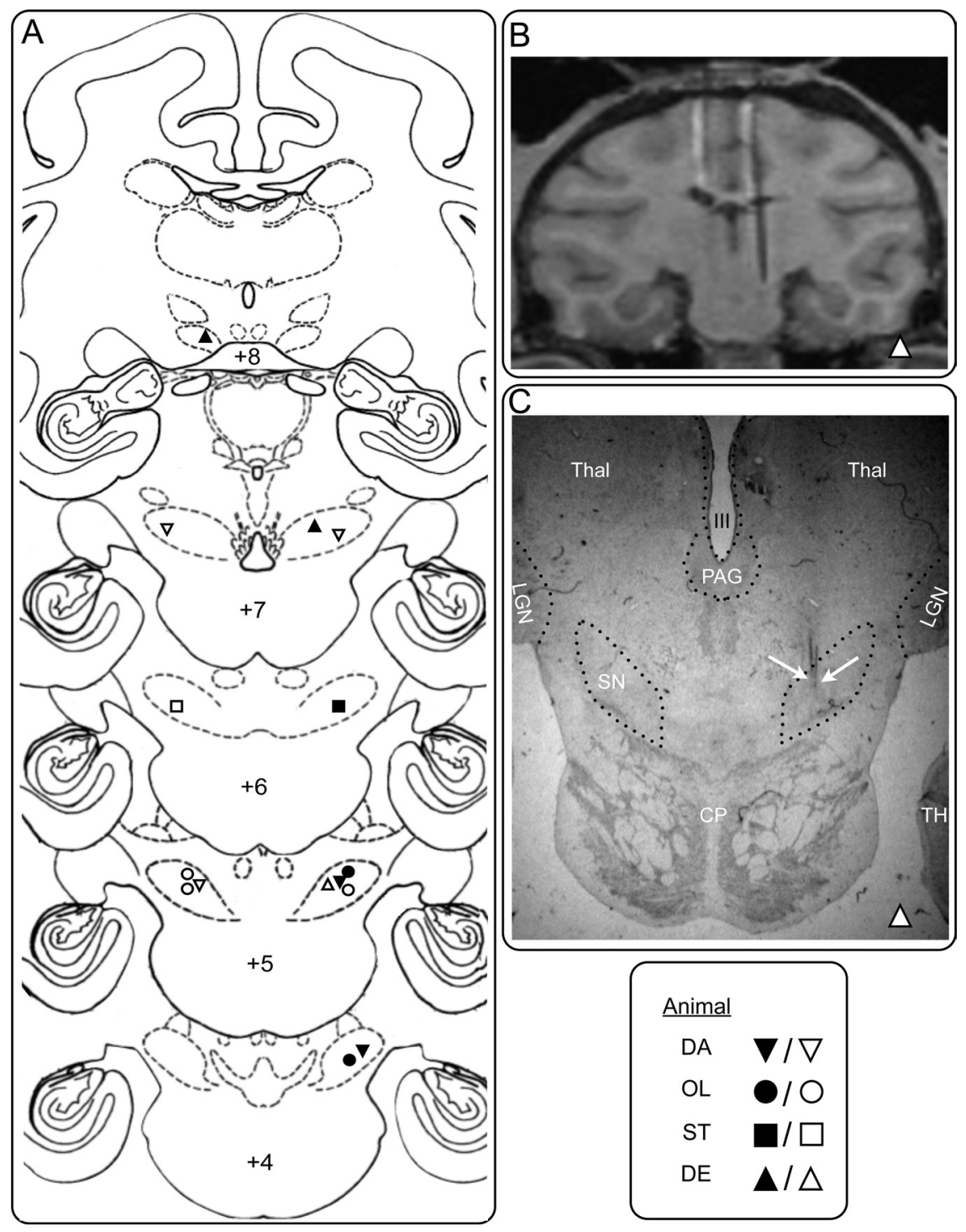

Figure 2. Histological verification of infusion sites in SNpr. $\boldsymbol{A}$, Coronal sections from a standard macaque brain showing the placement of cannulae in SNpr. Numbers $(+8,+7,+6,+5,+4)$ indicate the distance in millimeters from the interaural plane. Geometrical symbols show the placement of infusion sites determined from MRI (animals DA, OL, ST) or histology (DE). Black symbols represent sites used to examine SNpr-DLSC interactions. Clear symbols represent additional sites from which the described motor behaviors were evoked. $\boldsymbol{B}, \mathrm{MRI}$ section through SNpr in animal DE, corresponding approximately to level +7 , showing an electrode positioned in the infusion site in SNpr. C, Photomicrograph of a histological section through SNpr in the same animal (DE) at a level corresponding to the MRI section in $B(\sim+7)$. White arrows on the right point to traces of the cannula in the site corresponding to the electrode in $\boldsymbol{B}$. LGN, Lateral geniculate nucleus; Thal, thalamus; $S N$, substantia nigra pars reticulata; $C P$, cerebral peduncle; PAG, periaqueductal gray; III; third ventricle, TH; area TH of the parahippocampal cortex. Dotted lines show anatomical boundaries.

16 min following the onset of the head tilt), the rotational collis reached maximum severity: the head tilt extended over the shoulder, typically resulting in leaning of the body to the side of the head tilt. Thus, the presence of the body lean reflects the most severe manifestation of the torticollis. We recorded and analyzed the head tilt as a motor characteristic separate from the body lean. Head tilt and body lean were never observed during baseline conditions or following vehicle (saline) infusions in SNpr.

\section{Other behaviors evoked from SNpr}

In animals DE and DA, microinfusions of MUS in SNpr evoked rapid onset (latency to onset between 0 and $30 \mathrm{~min}$ postinfusion) contralaterally directed quadrupedal rotations.
In one animal (OL), choreiform dyskinesias were evoked following microinfusion of MUS in SNpr (Table 1). These responses were evoked from infusion sites distinct from those that evoked CD.

A quadrupedal rotation was defined as one complete rotation on all four limbs, directed toward the untreated side (i.e., left hemisphere SNpr infusions of MUS provoked clockwise rotation). As compared to baseline conditions, saline infusions did not significantly alter the amount of rotational behavior observed.

The dyskinesias evoked in animal OL were repetitive choreiform dyskinetic movements of both the contralateral arm and contralateral leg. These consisted of twisting and turning of lower and upper extremities in a circular manner, as we have described previously (Dybdal et al., 2012). Dyskinesias were not seen during baseline conditions or after saline infusions.

\section{Effect of MUS in DLSC}

Unilateral MUS infusions in the right or left DLSC were without significant effect on posture or locomotion.

\section{Intracerebral infusions to examine SNpr-DLSC interaction}

We examined the effect of MUS pretreatment using both ipsilateral and bilateral infusions in DLSC. The results from unilateral and bilateral pretreatments were not significantly different from each other and were therefore combined for statistical analysis. We also examined the effect of MUS pretreatment using contralateral infusions in DLSC; contralateral inhibition was without effect on the responses evoked from SNpr.

\section{Pretreatment of DLSC with MUS}

attenuated head tilt evoked from SNpr

Pretreatment of DLSC with MUS attenuated the head tilt (both laterocollis and rotational collis) evoked by unilateral infusion of MUS in SNpr. Head tilt was completely blocked by pretreatment of DLSC in three of the four animals. The effects of pretreatment of DLSC with MUS on head tilt are shown in Figure 3. As revealed by repeated measures one-way ANOVA, there was a main effect of drug treatment $\left(F_{(3,9)}=17.03 ; p<0.01\right)$. Holm-Sidak post hoc test (one-tailed) showed a significant decrease in head tilt when pretreatment of DLSC with MUS was compared to pretreatment of DLSC with saline $(p<0.04)$. Unilateral MUS in SNpr, with or without pretreatment of DLSC with saline, was significantly different from the baseline condition $(p<0.01)$. MUS in DLSC alone did not modify behavior, as compared to baseline conditions. 
Table 1. Profile of abnormal behaviors evoked by unilateral MUS in SNpr in each of four monkeys

\begin{tabular}{lllll}
\hline Monkey & Head tilt & Body lean & Quadrupedal rotation & Choreiform dyskinesia \\
\hline DE & + & + & + & - \\
DA & + & + & + & - \\
ST & + & + & - & - \\
OL & + & + & - & + \\
\hline
\end{tabular}

+ , Behavior was observed; - , behavior was not observed.

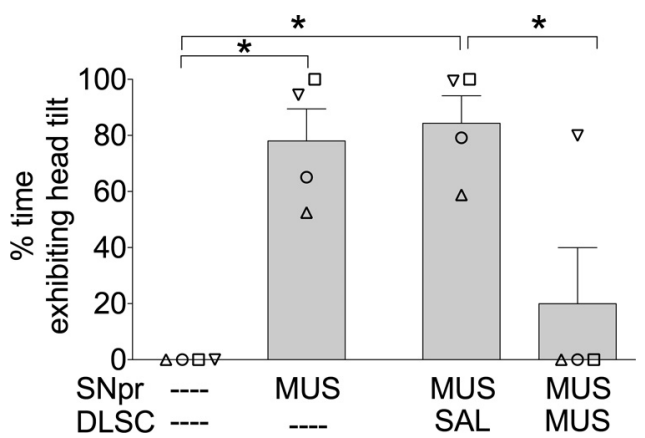

Figure 3. Pretreatment of DLSC with MUS attenuated head tilt evoked by MUS infusion in SNpr. Data are presented as mean (+SEM); individual symbols represent responses for each animal (symbols are identical to those presented in Figs. 1 and 2). Time of observation shown, $0-30$ min; ${ }^{*} p<0.05$.

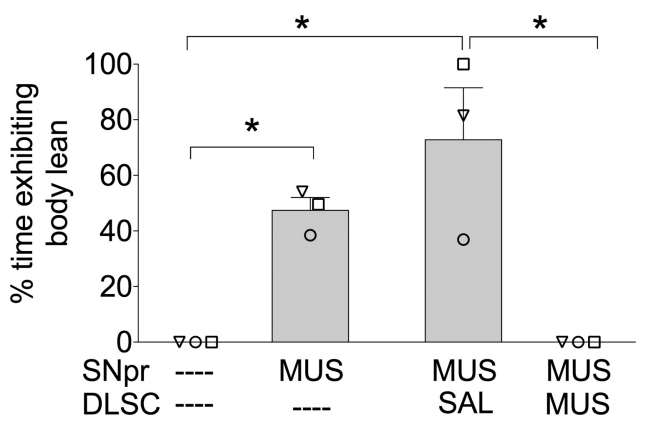

Figure 4. Pretreatment of DLSC with MUS attenuated body lean evoked by MUS infusion in SNpr. Data are presented as mean (+SEM); individual symbols represent responses for each animal (symbols are identical to those presented in Figs. 1 and 2) Time of observation, $0-30$ $\min ;{ }^{*} p<0.05$.

\section{Pretreatment of DLSC with MUS blocked body lean evoked by MUS in SNpr}

Three of the four animals (DA, ST, and OL) exhibited body lean after unilateral MUS in SNpr. This abnormality was completely blocked in all three animals after MUS pretreatment in DLSC. The effects of pretreatment of DLSC with MUS on body lean are shown in Figure 4. As revealed by repeated measures one-way ANOVA, there was a main effect of drug treatment $\left(F_{(3,6)}=\right.$ $16.192 ; p<0.05)$. Holm-Sidak post hoc test (one-tailed) showed a significant decrease in body lean when pretreatment of DLSC with MUS was compared to pretreatment of DLSC with saline $(p<0.003)$. Unilateral MUS in SNpr with or without pretreatment of DLSC with saline was significantly different than the baseline condition $(p<0.003)$.

\section{Pretreatment of DLSC with MUS did not alter SNpr-evoked quadrupedal rotation}

Unilateral microinfusions of MUS in SNpr evoked quadrupedal rotations in two of the four animals (DE and DA). To evaluate the effect of MUS in DLSC on this behavior, we pretreated the DLSC with MUS
Table 2. Effect of pretreatment with bilateral MUS in DLSC on the rate and peak of SNpr-evoked quadrupedal rotations

\begin{tabular}{|c|c|c|c|c|c|c|}
\hline \multirow[b]{2}{*}{ Monkey } & \multicolumn{2}{|c|}{ Baseline } & \multicolumn{2}{|c|}{ MUS in SNpr } & \multicolumn{2}{|c|}{$\begin{array}{l}\text { MUS in DLSC + } \\
\text { MUS in SNpr }\end{array}$} \\
\hline & Rate $^{a}$ & Peak ${ }^{b}$ & Rate $^{a}$ & Peak $^{b}$ & Rate $^{a}$ & Peak $^{b}$ \\
\hline $\mathrm{DE}$ & 2 & 0.1 & 2.6 & 40 & 5.4 & 81.3 \\
\hline DA & 1 & 0.1 & 2.6 & 37 & 3.3 & 49 \\
\hline
\end{tabular}

${ }^{a}$ Average rate per minute during the first 30 min of observation.

${ }^{b}$ Average peak during the first 30 min of observation.

Table 3. Abnormal behaviors evoked by unilateral BIC in DLSC ${ }^{a}$

\begin{tabular}{|c|c|c|c|c|c|}
\hline \multirow[b]{2}{*}{ Monkey } & \multicolumn{2}{|c|}{$\begin{array}{l}\text { Myoclonic } \\
\text { Movements }\end{array}$} & \multirow{2}{*}{$\begin{array}{l}\text { Contralateral eye } \\
\text { movements }\end{array}$} & \multirow{2}{*}{$\begin{array}{l}\text { Lip/mouth } \\
\text { twitch }\end{array}$} & \multirow{2}{*}{$\begin{array}{l}\text { Postural } \\
\text { asymmetry }\end{array}$} \\
\hline & Arm & Leg & & & \\
\hline$D E$ & 44 & 39 & 931 & 666 & $100 \%$ \\
\hline DA & 0 & 343 & 479 & 273 & $100 \%$ \\
\hline ST & 0 & 0 & 49 & 0 & $86 \%$ \\
\hline OL & 0 & 0 & 775 & 0 & $97 \%$ \\
\hline
\end{tabular}

${ }^{a}$ Total number of events and percent duration of state behavior (postural asymmetry) recorded over a 30 min observation period.

bilaterally before intranigral MUS. Bilateral treatment was used to avoid causing postural bias as a result of the pretreatment alone.

As shown in Table 2, bilateral pretreatment with MUS in DLSC did not modify the rate or peak total number of SNprevoked quadrupedal rotations. However, pretreatment with MUS in DLSC did modify the turning radius observed in subject $\mathrm{DE}$, resulting in a wider radius and less postural curvature than was observed after MUS in SNpr in the absence of MUS in DLSC.

\section{Abnormal postures and motor movements evoked by activation of DLSC with BIC}

Because our results with inhibition of DLSC indicated that activity in DLSC was necessary for the CD evoked from SNpr, we examined whether dystonic symptoms could be evoked by direct disinhibition of DLSC. We used unilateral BIC microinfusions into DLSC to block $\mathrm{GABA}_{\mathrm{A}}$ receptors and examined the behavioral responses to this treatment.

BIC microinfused into DLSC in two animals (DE and DA) evoked rapid onset ( $<30 \mathrm{~min}$ ) jerking movements of the contralateral leg (Table 3). These movements, which were intermittent, appeared to resemble myoclonic twitches, with flicking of the leg and foot. In one monkey (DE), arm movements were evoked from DLSC. The movements consisted of flicking of the arm downward toward the bottom of the cage. In addition, DE and DA exhibited twitching movements of the lip that selectively involved the upper lip on the side of the face contralateral to the infusion.

As our infusions were in the vicinity of sites where activation was previously reported to evoke contraversive saccadic eye movements (Hikosaka and Wurtz, 1985a), we measured eye movements after bicuculline microinfusions in DLSC. As shown in Table 3, in all animals tested, microinfusions of BIC evoked contralaterally directed eye movements with a rapid onset (within 10 min from the start of infusion, while the animal was still in the primate chair). A single eye movement was defined as a shift in the location of both eyes simultaneously to the side opposite to the infusion. The head was observed to turn in the same direction as the eye movements.

No signs of dystonic postures or movements were observed in any of the animals following the infusion of BIC in DLSC. 


\section{Discussion}

Our data support the hypothesis that DLSC mediates CD evoked by inhibition of SNpr. Both the dystonic head tilt and body lean evoked by muscimol in SNpr were significantly attenuated by DLSC inhibition. This suggests that activation of the DLSC is necessary for the emergence of the CD triggered by loss of SNpr output activity. Our results are the first to demonstrate a role of the DLSC in the expression of dystonic motor movements and posture and to reveal a functional relationship between SNpr and DLSC in mediating posture and movement in the nonhuman primate.

Our observation that CD evoked by inhibition of the central SNpr depends upon disinhibition of DLSC is analogous to the finding of Hikosaka and Wurtz (1983a,b, 1985a,b) that saccadic eye movements evoked by inhibition of SNpr are mediated by disinhibition of DLSC. In their studies, saccadic eye movements were associated with changes in activity only within the dorsolateral SNpr (within or adjacent to substantia nigra pars lateralis) (Hikosaka and Wurtz, 1985b) and not in the region from which we evoked CD (compare central electrode penetration in Fig. 3C in Hikosaka and Wurtz, 1983a with our cannula placement in Fig. 2). Consistent with this, we did not observe an increase in contraversive eye movements in association with the induction of CD.

Our data indicate that although DLSC is a crucial relay for the expression of CD evoked from SNpr, direct pharmacological activation of this region is not sufficient to evoke CD. We found that disinhibition of DLSC did not evoke abnormalities equivalent to those evoked by SNpr inhibition. Instead, we observed myoclonic twitching and flicking leg movements; these types of movements were not observed after muscimol infusions into SNpr. However, the pattern of DLSC activation resulting from the suppression of nigrotectal inhibition may not be accurately reproduced by focal drug application into DLSC. Consequently, these observations do not rule out the possibility that CD could originate in DLSC. In addition, it is possible that the combined disinhibition of DLSC plus an additional SNpr projection target may be necessary for evoking CD.

Quadrupedal rotations were evoked after inhibition of SNpr in two of the four animals in our study, from sites that were distinct from those that induced dystonia. This provided us the opportunity to determine whether inhibition of DLSC could also attenuate this behavior. We found that the quadrupedal rotations were not significantly affected by DLSC inhibition, suggesting that this behavioral response is dissociable from the SNpr-evoked CD. It is possible that our muscimol infusions in DLSC may have been located in regions mediating $\mathrm{CD}$, while rotational behaviors may depend upon regions of DLSC not reached by these infusions. It is also possible that the DLSC may not be required for the rotational behavior evoked by SNpr inhibition, as suggested by studies in rats (Di Chiara et al., 1982; Kilpatrick et al., 1982).

Previous studies have shown that CD can be evoked by inhibition in SNpr (Burbaud et al., 1998; Dybdal et al., 2012), and by activation of the putamen (Yoshida et al., 1991; Yamada et al., 1995). Inhibition of GPi did not evoke CD (Mink and Thach, 1991; Burbaud et al., 1998; Rolland et al., 2011), although activation of GPi induced dystonic postures without CD (Burbaud et al., 1998). Thus, as previously suggested (Guehl et al., 2009), CD is likely to be associated with increased activity in striatum, which in turn triggers inhibition in SNpr. Our results extend this circuitry to the DLSC by showing that inhibition of the nigrotectal pathway gives rise to CD. No similar direct projection to DLSC originates in GPi, which may explain why inhibition of GPi does not trigger CD. In contrast, quadrupedal rotation, a behavior that we found not to be mediated by DLSC, can be evoked by muscimol in GPi (Mink and Thach, 1991). Thus, these observations, taken together, suggest that whereas quadrupedal rotation evoked from GPi and SNpr may be mediated by thalamus and/or pedunculopontine nucleus, $\mathrm{CD}$ is dependent upon nigrotectal projections.

Deep brain stimulation (DBS) in GPi has been found effective for treating generalized dystonia (Cersosimo et al., 2009; Wolters, 2009; Welter et al., 2010; Kim et al., 2011) and, more recently, for treating CD (Goto and Yamada, 2004; Hung et al., 2007; Loher et al., 2008; Skogseid et al., 2012). Although the pathways mediating this therapeutic effect are not known, it has been suggested that DBS in GPi attenuates dystonia by modulating thalamocortical pathways (Wichmann and Delong, 2006). Identification of the relevant pathways is complicated by the fact that maximum improvement is seen only after several months of stimulation, suggesting that slow-onset neuroplastic changes are necessary; these could include changes in structures distant from the site of stimulation. Our data suggest that, unlike manipulations in GPi, manipulations of the nigrotectal pathway rapidly control the genesis of CD. Thus, for this specific type of focal dystonia, the nigrotectal pathway should be examined as a possible target for therapeutic activation.

\section{References}

Allen TA, Narayanan NS, Kholodar-Smith DB, Zhao Y, Laubach M, Brown TH (2008) Imaging the spread of reversible brain inactivations using fluorescent muscimol. J Neurosci Methods 171:30-38. CrossRef Medline Beckstead RM, Frankfurter A (1982) The distribution and some morphological features of substantia nigra neurons that project to the thalamus, superior colliculus and pedunculopontine nucleus in the monkey. Neuroscience 7:2377-2388. CrossRef Medline

Beckstead RM, Edwards SB, Frankfurter A (1981) A comparison of the intranigral distribution of nigrotectal neurons labeled with horseradish peroxidase in the monkey, cat, and rat. J Neurosci 1:121-125. Medline

Blandini F, Nappi G, Tassorelli C, Martignoni E (2000) Functional changes of the basal ganglia circuitry in Parkinson's disease. Prog Neurobiol 62:63-88. CrossRef Medline

Burbaud P, Bonnet B, Guehl D, Lagueny A, Bioulac B (1998) Movement disorders induced by gamma-aminobutyric agonist and antagonist injections into the internal globus pallidus and substantia nigra pars reticulata of the monkey. Brain Res 780:102-107. CrossRef Medline

Cersosimo MG, Raina GB, Benarroch EE, Piedimonte F, Alemán GG, Micheli FE (2009) Micro lesion effect of the globus pallidus internus and outcome with deep brain stimulation in patients with Parkinson disease and dystonia. Mov Disord 24:1488-1493. CrossRef Medline

Dean P, Redgrave P, Eastwood L (1982) Suppression of apomorphineinduced oral stereotypy in rats by microinjection of muscimol into midbrain. Life Sci 30:2171-2179. CrossRef Medline

Di Chiara G, Morelli M, Imperato A, Porceddu ML (1982) A re-evaluation of the role of superior colliculus in turning behaviour. Brain Res 237:61-77. CrossRef Medline

Dybdal D, Forcelli PA, Dubach M, Oppedisano M, Holmes A, Malkova L, Gale K (2012) Topography of dyskinesias and torticollis evoked by inhibition of substantia nigra pars reticulata. Mov Disord, in press.

Goto S, Yamada K (2004) Long term continuous bilateral pallidal stimulation produces stimulation independent relief of cervical dystonia. J Neurol Neurosurg Psychiatr 75:1506-1507. CrossRef Medline

Guehl D, Cuny E, Ghorayeb I, Michelet T, Bioulac B, Burbaud P (2009) Primate models of dystonia. Prog Neurobiol 87:118-131. CrossRef Medline

Gunne LM, Bachus SE, Gale K (1988) Oral movements induced by interference with nigral GABA neurotransmission: relationship to tardive dyskinesias. Exp Neurol 100:459-469. CrossRef Medline

Hikosaka O, Wurtz RH (1983a) Visual and oculomotor functions of monkey substantia nigra pars reticulata. I. Relation of visual and auditory responses to saccades. J Neurophysiol 49:1230-1253. Medline 
Hikosaka O, Wurtz RH (1983b) Visual and oculomotor functions of monkey substantia nigra pars reticulata. II. Visual responses related to fixation of gaze. J Neurophysiol 49:1254-1267. Medline

Hikosaka O, Wurtz RH (1985a) Modification of saccadic eye movements by GABA-related substances. I. Effect of muscimol and bicuculline in monkey superior colliculus. J Neurophysiol 53:266-291. Medline

Hikosaka O, Wurtz RH (1985b) Modification of saccadic eye movements by GABA-related substances. II. Effects of muscimol in monkey substantia nigra pars reticulata. J Neurophysiol 53:292-308. Medline

Hung SW, Hamani C, Lozano AM, Poon YY, Piboolnurak P, Miyasaki JM, Lang AE, Dostrovsky JO, Hutchison WD, Moro E (2007) Long-term outcome of bilateral pallidal deep brain stimulation for primary cervical dystonia. Neurology 68:457-459. CrossRef Medline

Kilpatrick IC, Collingridge GL, Starr MS (1982) Evidence for the participation of nigrotectal gamma-aminobutyrate-containing neurones in striatal and nigral-derived circling in the rat. Neuroscience 7:207-222. CrossRef Medline

Kim JP, Chang WS, Park YS, Chang JW (2011) Bilateral globus pallidus internus deep brain stimulation for DYT1 + generalized dystonia with previously received bilateral thalamotomy and unilateral pallidotomy. Stereotact Funct Neurosurg 89:205-209. CrossRef Medline

Laursen AM (1955) An experimental study of pathways from the basal ganglia. J Comp Neurol 102:1-25. CrossRef Medline

Loher TJ, Capelle HH, Kaelin-Lang A, Weber S, Weigel R, Burgunder JM, Krauss JK (2008) Deep brain stimulation for dystonia: outcome at longterm follow-up. J Neurol 255:881-884. CrossRef Medline

Malouin F, Bédard PJ (1983) Evaluation of head motility and posture in cats with horizontal torticollis. Exp Neurol 81:559-570. CrossRef Medline

Martin JH (1991) Autoradiographic estimation of the extent of reversible inactivation produced by microinjection of lidocaine and muscimol in the rat. Neurosci Lett 127:160-164. CrossRef Medline

Martin JH, Ghez C (1999) Pharmacological inactivation in the analysis of the central control of movement. J Neurosci Methods 86:145-159. CrossRef Medline

Mink JW (2003) The Basal Ganglia and involuntary movements: impaired inhibition of competing motor patterns. Arch Neurol 60:1365-1368. CrossRef Medline

Mink JW, Thach WT (1991) Basal ganglia motor control. III. Pallidal ablation: normal reaction time, muscle cocontraction, and slow movement. J Neurophysiol 65:330-351. Medline

Nauta WJ, Mehler WR (1966) Projections of the lentiform nucleus in the monkey. Brain Res 1:3-42. CrossRef Medline
Niemi-Junkola UJ, Westby GW (1998) Spatial variation in the effects of inactivation of substantia nigra on neuronal activity in rat superior colliculus. Neurosci Lett 241:175-179. CrossRef Medline

Redgrave P, Marrow L, Dean P (1992) Topographical organization of the nigrotectal projection in rat: evidence for segregated channels. Neuroscience 50:571-595. CrossRef Medline

Rolland AS, Karachi C, Muriel MP, Hirsch EC, François C (2011) Internal pallidum and substantia nigra control different parts of the mesopontine reticular formation in primate. Mov Disord 26:1648-1656. CrossRef Medline

Sidibé M, Bevan MD, Bolam JP, Smith Y (1997) Efferent connections of the internal globus pallidus in the squirrel monkey: I. Topography and synaptic organization of the pallidothalamic projection. J Comp Neurol 382:323-347. CrossRef Medline

Skogseid IM, Ramm-Pettersen J, Volkmann J, Kerty E, Dietrichs E, Røste GK (2012) Good long-term efficacy of pallidal stimulation in cervical dystonia: a prospective, observer-blinded study. Eur J Neurol 19:610-615. CrossRef Medline

Taha EB, Dean P, Redgrave P (1982) Oral behaviour induced by intranigral muscimol is unaffected by haloperidol but abolished by large lesions of superior colliculus. Psychopharmacology 77:272-278. CrossRef Medline

Wellman LL, Gale K, Malkova L (2005) GABAA-mediated inhibition of basolateral amygdala blocks reward devaluation in macaques. J Neurosci 25:4577-4586. CrossRef Medline

Welter ML, Grabli D, Vidailhet M (2010) Deep brain stimulation for hyperkinetics disorders: dystonia, tardive dyskinesia, and tics. Curr Opin Neurol 23:420-425. Medline

West EA, DesJardin JT, Gale K, Malkova L (2011) Transient inactivation of orbitofrontal cortex blocks reinforcer devaluation in macaques. J Neurosci 31:15128-15135. CrossRef Medline

Wichmann T, Delong MR (2006) Deep brain stimulation for neurologic and neuropsychiatric disorders. Neuron 52:197-204. CrossRef Medline

Wolters A (2009) Long-term efficacy of deep brain stimulation in patients with dystonia (in German). Fortschr Neurol Psychiatr 77 [Suppl 1]:S61-S63.

Yamada H, Fujimoto K, Yoshida M (1995) Neuronal mechanism underlying dystonia induced by bicuculline injection into the putamen of the cat. Brain Res 677:333-336. CrossRef Medline

Yoshida M, Nagatsuka Y, Muramatsu S, Niijima K (1991) Differential roles of the caudate nucleus and putamen in motor behavior of the cat as investigated by local injection of GABA antagonists. Neurosci Res 10:3451. CrossRef Medline 